Nuclear reactions used for superheavy element research

M. A. Stoyer

February 27, 2008

Compound Nuclear Reactions and Related Topics Fish Camp, CA, United States October 22, 2007 through October 26, 2007 
This document was prepared as an account of work sponsored by an agency of the United States government. Neither the United States government nor Lawrence Livermore National Security, LLC, nor any of their employees makes any warranty, expressed or implied, or assumes any legal liability or responsibility for the accuracy, completeness, or usefulness of any information, apparatus, product, or process disclosed, or represents that its use would not infringe privately owned rights. Reference herein to any specific commercial product, process, or service by trade name, trademark, manufacturer, or otherwise does not necessarily constitute or imply its endorsement, recommendation, or favoring by the United States government or Lawrence Livermore National Security, LLC. The views and opinions of authors expressed herein do not necessarily state or reflect those of the United States government or Lawrence Livermore National Security, LLC, and shall not be used for advertising or product endorsement purposes. 


\title{
Nuclear Reactions Used For Superheavy Element Research
}

\author{
Mark A. Stoyer \\ N Division, Physical Sciences Directorate, Lawrence Livermore National Laboratory, Livermore CA 94550 USA
}

\begin{abstract}
Some of the most fascinating questions about the limits of nuclear stability are confronted in the heaviest nuclei. How many more new elements can be synthesized? What are the nuclear and chemical properties of these exotic nuclei? Does the "Island of Stability" exist and can we ever explore the isotopes inhabiting that nuclear region? This paper will focus on the current experimental research on the synthesis and characterization of superheavy nuclei with $\mathrm{Z}>112$ from the Dubna/Livermore collaboration. Reactions using ${ }^{48} \mathrm{Ca}$ projectiles from the U400 cyclotron and actinide targets $\left({ }^{233,238} \mathrm{U},{ }^{237} \mathrm{~Np},{ }^{242,244} \mathrm{Pu},{ }^{243} \mathrm{Am},{ }^{245,248} \mathrm{Cm},{ }^{249} \mathrm{Cf}\right)$ have been investigated using the Dubna Gas Filled Recoil Separator in Dubna over the last 8 years. In addition, several experiments have been performed to investigate the chemical properties of some of the observed longer-lived isotopes produced in these reactions. Some comments will be made on nuclear reactions used for the production of the heaviest elements. A summary of the current status of the upper end of the chart of nuclides will be presented.
\end{abstract}

Keywords: Superheavy Elements, Nuclear reactions

PACS: 25.70.Gh, 27.90.+b

\section{INTRODUCTION}

In this paper, some research highlights in the superheavy element field, including both physics and chemistry work, will be presented and the current status of research in this exciting area of science will be indicated. Indeed, over the past 8-9 years, some remarkable progress has been made in investigating the heaviest elements, including the discovery of several new elements. For a more complete discussion of any of the topics mentioned in this paper, please refer to the references provided.

A variety of experimental techniques have been used to make new chemical elements, including heavy-ion transfer reactions, cold- or hot-fusion evaporation reactions, neutron captures, and light-ion charged particle-induced reactions. In a heavy-ion transfer reaction, typically, a heavy nucleus hits a target nucleus and transfers one or more (generally a few) nucleons (protons or neutrons). A light-ion charged particle-induced reaction involves a light nucleus (like a deuteron, proton, or ${ }^{4} \mathrm{He}$ nucleus) hitting a target nucleus and transferring a particle with charge such as a proton or alpha particle. Neutron capture reactions involve a neutron hitting a target nucleus and being absorbed into that nucleus. All of these techniques involve transmuting one kind of nucleus into another. Two new elements, einsteinium (Es) and fermium (Fm), with $\mathrm{Z}=99$ and 100, respectively, were discovered in the debris of an atmospheric nuclear explosion. This entailed rapid capture of many neutrons by uranium, followed subsequently by up to about 20 beta decays of the extremely neutron-rich uranium isotope and its daughters to more stable elements, a process similar to r-process nucleosynthesis in some types of stars. Each of these techniques has advantages and disadvantages making them suitable for studying nuclei in certain regions.

Modern "nuclear alchemists" working on the synthesis of superheavy elements "transmute" one element into another using particle accelerators and smashing a beam of one element into a target of another element to produce the desired element. The types of nuclear reactions that have been successfully used to produce new elements in the last decade or so are cold-fusion reactions and hot-fusion reactions.

Cold-fusion reactions use more symmetric beam and target nuclei, produce a compound nucleus with generally lower excitation energy that typically requires evaporation of one or no neutrons, and generate less neutron-rich isotopes of an element. They have higher survival probabilities with respect to fission but have lower fusion probabilities. An example of this type of reaction is ${ }^{70} \mathrm{Zn}+{ }^{208} \mathrm{~Pb} \rightarrow$ ${ }^{277} 112+1 \mathrm{n}$ with a cross-section of about 1 picobarn $\left(10^{-36} \mathrm{~cm}^{2}\right)$. Because the 112 isotope ultimately decays by alpha emission to known nuclei, namely, isotopes of elements 102 (nobelium) and 104 (rutherfordium), identification of this element is straightforward. 
Hot-fusion reactions use more asymmetric beam and target nuclei, produce a compound nucleus with generally higher excitation energy that typically requires evaporation of three to five neutrons, and generate more neutron-rich isotopes of an element. They have lower survival probabilities with respect to fission but have higher fusion probabilities. An example of this type of reaction is ${ }^{48} \mathrm{Ca}+{ }^{244} \mathrm{Pu} \rightarrow$ ${ }^{288} 114+4 \mathrm{n}$, also with a cross section of about $1 \mathrm{pb}$. Because of the neutron richness of this isotope of element 114, it never subsequently decays to any known isotope, and thus its identification is more problematic.

Both of these types of reactions utilize doubly magic nuclei, as either target or projectile to attempt to increase the stability of the compound nucleus. Coldfusion reactions have been successful in producing elements 104-112, and hot-fusion reactions have recently provided evidence for elements 113-116 and 118 (Fig. 1).

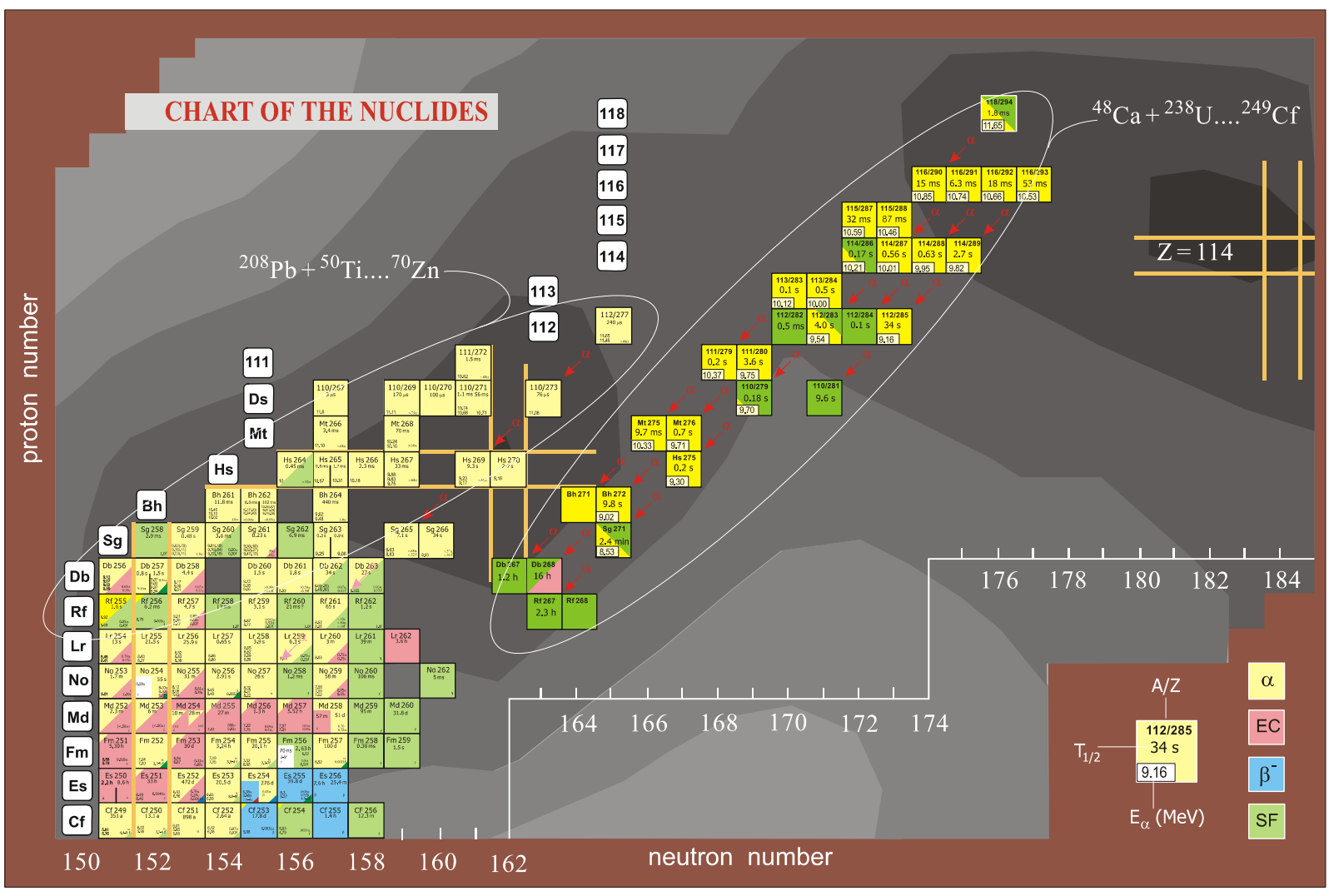

FIGURE 1. Upper end of the chart of nuclides as of about 2004. The nuclides produced by cold fusion reactions are shown in the ellipse at the left, and those produced by hot fusion reactions are shown at the right. Both reactions take advantage of doubly magic nuclei and the special stability provided by either the target (cold fusion) or projectile (hot fusion). Several nuclides have been added in the intervening years, including directly produced isotopes of element 113 by both Dubna and RIKEN.

Once the desired reaction has been performed, scientists then use sophisticated separators and detectors to observe the presence of the newly formed nuclide. Several types of separators can be utilized for this, including velocity filters such as VASSALISSA at Dubna and SHIP at GSI, and gas-filled separators like Dubna Gas Filled Recoil Separator (DGFRS) at Dubna and BGS at Berkeley. These separators provide a very large suppression of unwanted reaction products, transfer products, and beam-like particles. Typical detection involves positions sensitive particle detectors where the implant of the recoiling nuclide that has passed through the separator is observed, and then subsequent alpha and spontaneous fission (SF) decays are correlated in position. The energies of the decays and decay times are measured using such a system. The DGFRS is shown in Fig. 2. 


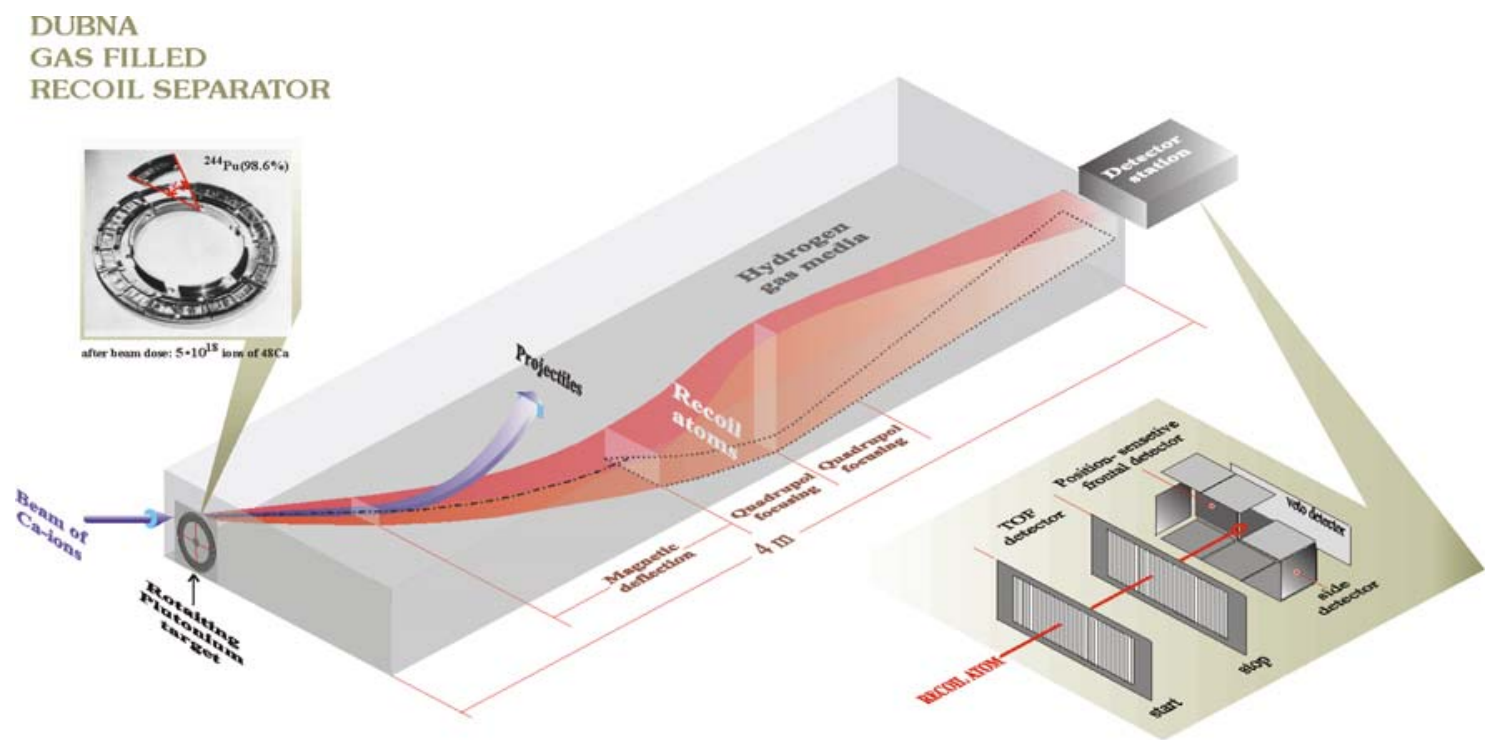

Yuri Oganessian FLNR (JNRR) June 14, 1999

FIGURE 2. A schematic of the Dubna Gas Filled Recoil Separator (DGFRS) showing the rotating target, separator and position sensitive detector system. This separator has been used to investigate the production of a variety of superheavy elements.

\section{EXPERIMENTS BY DUBNA/LIVERMORE COLLABORATION ON DGFRS ON ELEMENTS 113, 114, 115, 116 AND 118}

Reactions using ${ }^{48} \mathrm{Ca}$ projectiles from the $\mathrm{U} 400$ cyclotron and actinide targets $\left({ }^{233,238} \mathrm{U},{ }^{237} \mathrm{~Np},{ }^{242,244} \mathrm{Pu}\right.$, ${ }^{243} \mathrm{Am},{ }^{245,248} \mathrm{Cm},{ }^{249} \mathrm{Cf}$ ) have been investigated using the Dubna Gas Filled Recoil Separator in Dubna over the last 8 years. ${ }^{1-4}$ The 34 new nuclides that have been observed are indicated in Fig. 1 (right hand ellipse). Nuclear decay properties were measured and some isotopes with significantly long half lives were observed. A review of all the Dubna/Livermore experiments may be found in Ref. [5]. Interestingly, while detailed excitation function measurements have been performed for nearly all of the produced nuclides, the peak cross-sections for production of elements 112-116 tend to be about $1 \mathrm{pb}$. An enhanced fission barrier and reactions with deformed target nuclei where the orientation may play a role, may be leading to the increased survivability of the produced isotopes.

\section{EXPERIMENTS BY RIKEN ON ELEMENT 113}

Cold fusion reaction studies have been done at the GARIS gas filled separator at RIKEN to produce two atoms of element 113 at the $78 \mathrm{fb}$ cross-section level. ${ }^{6}$ This represents many months of beam time and the current limit of cold-fusion reaction production of the heaviest elements. Work at RIKEN has also reproduced earlier element 112 work at GSI.

\section{EXPERIMENTS BY PSI/DUBNA COLLABORATION ON CHEMISTRY OF ELEMENT 112}

Gas-phased chemistry experiments utilizing the ${ }^{48} \mathrm{Ca}+{ }^{238} \mathrm{U}$ nuclear reaction to produce ${ }^{283} 112$ and thermal gradient column of an array of silicon detectors (half gold coated) to detect alpha and SF decays was used to indicate that element 112 behaves more mercury-like than noble-gas-like. ${ }^{7}$ Enthalpies of adsorption on the gold surface of the detectors were measured. 


\section{EXPERIMENTS BY DUBNA/LIVERMORE COLLABORATION ON CHEMISTRY OF DB - THE DECAY DESCENDENT OF ELEMENT 115}

Production of a long-lived isotope of $\mathrm{Db}$ was accomplished using the ${ }^{48} \mathrm{Ca}+{ }^{243} \mathrm{Am} \rightarrow{ }^{288} 115+3 \mathrm{n}$ reaction and allowing element 115 to decay by a series of 5 alpha decays to ${ }^{268} \mathrm{Db}$ that has a half life of roughly 1 day. ${ }^{8-10}$ This experiment utilizes a $\mathrm{Cu}$ catcher-block to collect all of the recoiling products (Fig. 3), and then several chemistries that eliminated any actinides and separated group 4 and group 5 elements, followed by counting of chemical fractions.

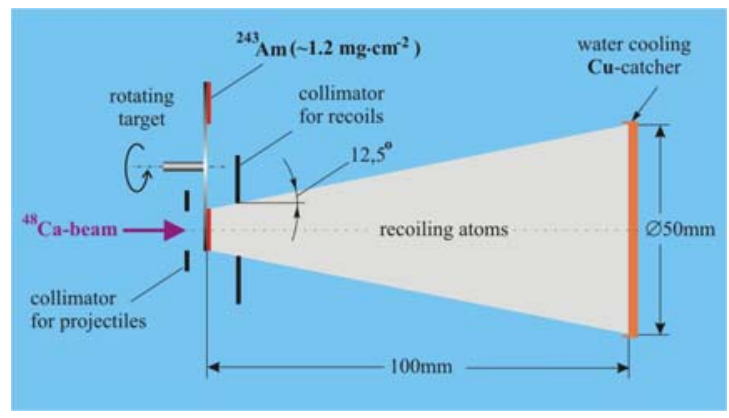

FIGURE 3. A schematic of the target and $\mathrm{Cu}-$ catcher used for the chemical study of element 112 . The un-utilized beam penetrated farther into the $\mathrm{Cu}$ catcher than the recoiling 115 atoms, thus permitting a physical separation when the surface of the $\mathrm{Cu}$-catcher was machined off once a day for chemistry.

A number of SF were observed in the course of several experiments. The LLNL developed chemistry used a reverse-phased column to provide intra-group separation of the group 5 elements. In the second experiment, $5 \mathrm{SF}$ events were observed in the group 5 fractions, three of which were in the Ta fractions, and no SF events were observed in the group 4 fractions or $\mathrm{Nb}$ fractions.

\section{EXPERIMENTS BY JYVÄSKYLÄ AND ANL ON DETAILED NUCLEAR SPECTROSCOPY OF ${ }^{254} \mathrm{NO}$}

Complete spectroscopy of ${ }^{254}$ No was performed by several groups and for the first time a $3^{+}$isomeric level whose configuration was identified as containing proton character based on the $2 f_{5 / 2}$ state from above the $Z=114$ shell gap was observed. ${ }^{11,12}$ The excited states of ${ }^{254}$ No were populated using the ${ }^{48} \mathrm{Ca}+{ }^{208} \mathrm{~Pb}$ nuclear reaction and decayed by emitting gamma-rays, $\mathrm{X}$ rays and electrons, conversion and Auger, to the ground state, which then decayed via alpha-emission. Conversion and Auger electrons defined a clean signature of isomeric decay, alpha emission established the decaying nucleus as ${ }^{254} \mathrm{No}$ and the conversion electrons and gamma-rays enabled the construction of the ${ }^{254}$ No nuclear level scheme. Since gamma radiation arises from electromagnetic effects, characterization of the electric or magnetic nature of a given gamma-ray, namely, gamma-ray multipolarity measurements, determined the spins and parities of many of the states in that level scheme and a magnetic moment measurement (g-factor) established one of the states as a two proton excitation. This structure is firmly established as a two-proton excitation involving the $2 f_{5 / 2}$ proton orbital from above the $\mathrm{Z}=114$ shell gap. This very nicely establishes the location of one proton single particle level which influences the superheavy element region and the "Island of Stability". In addition, preliminary estimates of the $\mathrm{Z}=114$ shell gap are on the order of $2 \mathrm{MeV}$.

\section{EXPERIMENTS BY GSI ON ${ }^{283} 112$}

Production of ${ }^{283} 112$ using the ${ }^{48} \mathrm{Ca}+{ }^{238} \mathrm{U}$ reaction was observed at GSI for the first time reproducing the results obtained at Dubna. ${ }^{13}$ The SHIP separator was used for separation of the element 112 recoils from the beam and unwanted reaction products. Two evaporation residue (EVR) -alpha-SF decay chains and two EVR-SF decay chains were observed in this experiment.

\section{DISCUSSION}

A comparison of the cross-sections obtained for ${ }^{48} \mathrm{Ca}$-induced hot fusion reactions shows the effects of initial orientation of the colliding nuclei and allows the extraction of fission barriers $B_{f}$ for both ${ }^{256} \mathrm{No}$ and ${ }^{292} 114$ as shown in Fig. 4. Surprisingly, the fission barrier is about 6-7 MeV for both nuclei, contributing to the enhanced survivability of the element 114 nucleus with respect to fission. Note the nuclear shape orientations for the ${ }^{48} \mathrm{Ca}+{ }^{244} \mathrm{Pu}$ reaction as indicated on the top of the figure, resulting in different excitation energies and cross-sections, also distinct from two spherical nuclei as shown in the left part of the figure. The shape of the $\Sigma \sigma_{x n}$ reaction cross-section is similar for both reactions, though the peak is 3-4 orders of magnitude lower for the actinide target and shifted much farther above the Bass barrier nearer to the equatorial orientation configuration. 


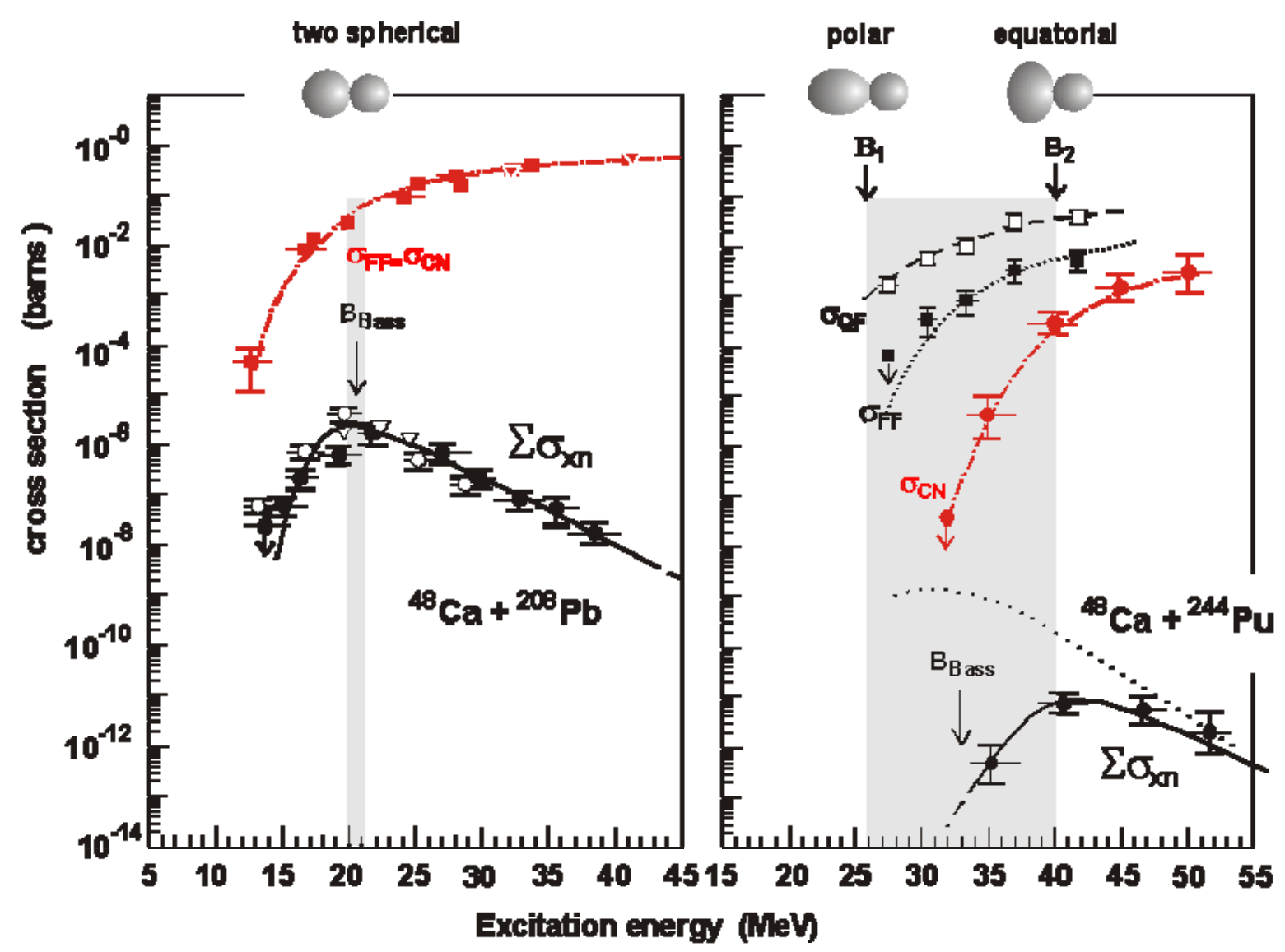

FIGURE 4. A comparison of the cross-sections for two ${ }^{48} \mathrm{Ca}$-induced reactions as a function of excitation energy. The dot-dashed curves are the compound nucleus formation cross-sections and the bottom solid curves are the final production cross-sections for all neutron-emission channels. The difference between these two curves is the fissility, which is nearly identical at $40 \mathrm{MeV}$ excitation energy (near the peak of the 4n-evaporation channel) for both reactions.

\section{CONCLUSIONS}

This paper has attempted to indicate the current state-of-affairs for the heaviest elements and highlight some of the exciting recent results in this area of science. The fascinating interplay between chemistry and physics investigations of these exotic man-made elements was shown. Some of the most fundamental questions about the production of the heaviest elements will be answered by continuing investigation into the nuclear reactions that produce them, and continuing to probe this fascinating region of the chart of nuclides.

\section{ACKNOWLEDGMENTS}

This work has been performed with the support of the Russian Ministry of Atomic Energy and grant of RFBR No. 04-02-17186. The ${ }^{249} \mathrm{Cf}$ target material was provided by the U.S. DOE through ORNL and partially by RIAR, Dimitrovgrad. The ${ }^{245} \mathrm{Cm}$ target material was provided by RFNC-VNIIEF, Sarov, Russia. The ${ }^{243} \mathrm{Am}$ target material was provided by RIAR, Dimitrovgrad, and by the U.S. DOE through ORNL. The ${ }^{244} \mathrm{Pu}$ target material was provided by the U.S. DOE through ORNL. Much of the support for the LLNL authors was provided through the U.S. DOE under Contract Nos. W-7405-Eng-48 and DE-AC5207NA27344. These studies were performed in the framework of the Russian Federation/U.S. Joint Coordinating Committee for Research on Fundamental Properties of Matter. 


\section{REFERENCES}

1. Yu. Ts. Oganessian, et al., Phys. Rev. Lett. 83, 3154 (1999).

2. Yu. Ts. Oganessian, et. al., Phys. Rev. C 69, 054607 (2004).

3. Yu. Ts. Oganessian, et al., Phys. Rev. C 69, 021601 (2004).

4. Yu. Ts. Oganessian, et al., Phys. Rev. C 74, 044602 (2006).

5. Yu. Ts. Oganessian J. Phys. G Nucl. Part. Phys. 34, R165 (2007).

6. K. Morita, et al., J. Phys. Soc. Japan 73, 2593 (2004).

7. R. Eichler, et al. in Proceedings of the IX International Conference on Nucleus Nucleus Collisions held Aug. 28 - Sep. 1, 2006 in Rio de Janiero, Brazil, Nucl. Phys. A. 787, 373c (2007).

8. S.N. Dmitriev, et al., Mend. Commun. 1, 1 (2005).

9. Yu. Ts. Oganessian, et al., Phys. Rev. C 72, 034611 (2005).

10. N.J. Stoyer, et al. in Proceedings of the IX International Conference on Nucleus Nucleus Collisions held Aug. 28 - Sep. 1, 2006 in Rio de Janiero, Brazil, Nucl. Phys. A. 787, 388c (2007).

11. R.-D. Herzberg, et al., Nature 442, 896 (2006).

12. S.K. Tandel, et al., Phys. Rev. Lett. 97, 082502 (2006).

13. S. Hofmann, et al., EPJA 32, 251 (2007).

This work performed under the auspices of the U.S. Department of Energy by Lawrence Livermore National Laboratory under Contract DE-AC52-07NA27344. 